\title{
Multidimensional internal dynamics underlying the perception of motion
}

\author{
Mark Wexler \\ Laboratoire Psychologie de la Perception \\ CNRS and Université Paris Descartes, France
}

February 19, 2018

\begin{abstract}
When ambiguous visual stimuli are presented continuously, they often lead to oscillations between usually two perceptions. Because of these oscillations, it has been thought that the underlying neural dynamics also arises from a binary or two-state system. Contradicting the binary assumption, it has been shown recently that the perception of some ambiguous stimuli is governed by continuously varying internal states, measured as biases that differ considerably from one observer to the next and that can also evolve over time (Wexler et al., 2015). Here I study bias patterns in the motion quartet, an ambiguous apparent motion stimulus, as the quartet's orientation is varied. The bias patterns are robustly idiosyncratic, and are even more complex than those that have been described previously. There are two qualitatively different bias types: some observers prefer a translation axis, while others show preference for a rotation direction. Each type also varies parametrically: the orientation of the preferred axis, and the direction of preferred rotation. The are also clear cases of combination of the two bias types. When measured repeatedly over 9 hours, the bias patterns usually remain stable, but also sometimes evolve both parametrically (e.g., change of preferred axis), as well as across bias type (change from axial to rotational bias). Control experiments revealed that the variety of bias patterns observed across subjects, and their changes over time, are not due to voluntary decisions. Overall, these results exhibit the multidimensional complexity of internal states underlying the perception of even simple stimuli.
\end{abstract}

\section{Introduction}

Reversible or bistable stimuli have long attracted attention because, in the absence of external cues that favor a particular interpretation, the dynamics of perceptual choice provides a uniquely sensitive probe of the neural mechanisms of perception (Necker, 1832; Wheatstone, 1838; Rubin, 1921; Wallach, 1935; Attneave, 1971; Leopold \& Logothetis, 1999; Blake \& Logothetis, 2002; Long \& Toppino, 2004; Sterzer, Kleinschmidt \& Rees, 2009). Such ambiguous stimuli have been used to study the mechanisms of adaptation, priming, context, history, memory, cue integration, cortical dynamics, to cite some exam- 
ples. It is often assumed that neural dynamics is isomorphic to overt perceptual dynamics. For example, if the perception of stimulus switches between two interpretations, then the underlying neural representations must also be binary. Here I show that this assumption underestimates the complexity of the underlying brain circuits. By measuring the population distribution and temporal evolution of the individual observers' biases in the perception of a well-known twofold-ambiguous stimulus-the motion quartet-I show that the biases reflect complex and multidimensional internal states.

The motion quartet (also called the "bistable quartet" or "stroboscopic alternative motion") is a two-frame ambiguous motion stimulus, illustrated in Figure 1a (Schiller, 1933; Gengerelli, 1948; Ramachandran \& Anstis, 1983b). In the first frame of the usual version of the quartet, two identical objects appear at opposite corners of an imaginary square; in the second frame, the same two objects appear in the other two corners. If the visual system matches each of the objects in the second frame to a single and unique object in the first frame, two apparent motions can be perceived, illustrated in Figures $1 \mathrm{~b}$ and c. Because the distances in the two solutions are the same, neither the slow-motion prior nor any other perceptual cue can disambiguate the two solutions. The motion quartet has been used to probe contextual and priming effects in motion perception (Ramachandran \& Anstis, 1983b; Ramachandran \& Anstis, 1983a; Anstis \& Ramachandran, 1987), hysteresis and sequence effects (Hock et al., 1993; Maloney et al., 2005), and analogies between visual and tactile motion perception (Carter, Konkle, et al., 2008; Liaci et al., 2016; Haladjian et al., n.d.), as well as to study the neural correlates of visual consciousness (Sterzer \& Kleinschmidt, 2007; Zhang et al., 2012) and the effects of neuroanatomy on motion perception (Genç et al., 2011; Shimono et al., 2012).

The subject of interindividual differences in vision has always elicited a certain amount of interest, going back to philosophers' questions about the subjective appearance of colors (Locke, 1690) and the "personal equation" in astronomy (Bessel, 1823), as well as more recently (Coren \& Porac, 1987; Wilmer, 2008; Grzeczkowski et al., 2017), including in the context of multistable stimuli (Kleinschmidt et al., 2012). Nevertheless, most visual psychophysics is carried out with the tacit assumption that the appearance of suprathreshold stimuli is invariant across observers, or that any variations are small and can be ignored. This assumption is probably the reason why the large individual differences in the appearance of The Dress-a photograph that spread on the internet in 2015-impressed not only naïve observers but psychophysicists as well (Witzel et al., 2017).

It has recently become clear that the perception of each observer is shaped by individual biases that can differ greatly from one observer to the next and that can persist over long periods of time (Carter \& Cavanagh, 2007; Afraz et al., 2010; Mamassian \& Wallace, 2010; Houlsby et al., 2013; Schütz, 2014; Wexler et al., 2015; Schütz \& Mamassian, 2016; Goutcher, 2016; Kosovicheva \& Whitney, 2017). For example, with my colleagues I have recently shown that the perception of two families of stimuli are shaped by individual biases that are a preferred 3D surface orientation for one of the families, and a preferred motion direction for the other family (Wexler et al., 2015). In an internet-based, large-sample experiment we showed that the biases differed greatly-in fact, sometimes maximally-from one observer to the next, the two biases were independent from one 
another within observers, and were quite stable over durations up to one year. However, a longitudinal experiment showed that the biases could undergo small changes (and sometimes large ones, although more rarely) over time, with dynamics that are at least partly described as random walks. We argued that these biases are persistent states of the brain, whose internal dynamics and interactions with external stimuli must be understood in order to have a complete description of perceptual processes (Wexler et al., 2015).

In the current study I measure individual biases in the perception of the motion quartet. These biases turn out to have novel and interesting properties. In the first of the two experiments described here, I measured the bias patterns for the motion quartet in a relatively large sample (106 subjects). Conventional studies of ambiguous stimuli like the motion quartet tend to concentrate on their bistable aspect and present the exact same stimulus either continuously or repeatedly with intervening blanks. In the current studies, on the other hand, the two-frame sequence was presented only once on each trial, and its overall orientation varied randomly from trial to trial. Not having witnessed spontaneous oscillations from repeated exposure to the same stimulus, many subjects did not realize that the stimuli are ambiguous, judging from their qualitative reports. In the second experiment, I measured the time evolution of the individual bias patterns by repeatedly measuring them ( 45 times) over a period of 9 hours.

To get a good feel for the stimulus, I urge the reader to try an on-line demonstration at http://lab-perception.org/demo/q. The demonstration is a briefer version of Experiment 1. Additionally, after the 24 trials (which should take no more than a minute or two to complete), the reader will be shown his or her own bias pattern, which can be compared to the data in this article.

\section{Experiment 1: Population sample of bias patterns}

This experiment measured bias patterns for the motion quartet in a fairly large sample of subjects $(N=106)$, at one point in time. The quartet was presented once ( 2 frames) on every trial, with its orientation varying randomly from trial to trial.

\subsection{Methods}

In order to obtain a large sample, this experiment was performed by subjects inside an internet browser on their own computers outside the laboratory. I used methods developed in an earlier study (Wexler et al., 2015) in order to control stimuli and experimental procedures as much as possible in this unconventional setting (see below for details). Similar results were obtained in a more traditional laboratory setting, including pilot studies for this experiment, as well as in the second experiment presented later in this article. 


\subsubsection{Stimuli}

Stimuli were displayed and responses collected in an internet browser, with the experiment programmed in Javascript and HTML5. Tablets and telephones were not allowed, in order to control monitor orientation. It was possible to measure video frame rate, which was above $30 \mathrm{~Hz}$ for all subjects. Subjects were encouraged to make the browser window full-screen or as large as possible, to minimize extraneous stimuli; the browser window filled at least $60 \%$ of the monitor's area in all cases but one (where it filled $47 \%$ ). Subjects were instructed to sit upright at a comfortable distance from the monitor. For further details, see Wexler et al. (2015).

Stimulus geometry will be given in pixels. It is impossible to translate from pixels to degrees of visual angle, because both pixel size and the distance between subject and monitor are unknown. Additionally, some systems with high-resolution monitors scale pixel units in order to make small text and images easier to see, so that a logical pixel may correspond to more than one physical pixel. However, at the subjects' median resolution of $1366 \times 768$, assuming a common 13 inch $16 / 9$ diagonal monitor viewed at a typical distance of $50 \mathrm{~cm}$, and no pixel scaling, 150 pixels (the distance from the center of the imaginary square to its corners) is about $3.3^{\circ}$ of visual angle.

The main stimulus consisted of two frames, each containing two disks lying on the opposite corners of an imaginary square (labeled 1 and (2) in Figure 1a). The disks had radii of 20 pixels, and were drawn in black on a medium gray background. They were drawn so that their centers were at a distance of 150 pixels from the center of the window. The entire figure (the imaginary square) was oriented at one of 24 angles: $3.75^{\circ}, 11.25^{\circ}$, $18.75^{\circ}, \ldots, 176.25^{\circ}$. (The orientations were between 0 and $180^{\circ}$ because adding $180^{\circ}$ to the stimulus orientation leaves the stimulus unchanged.) In addition to the disks, a white cross (30 pixels wide) was displayed in the center of the window, which the subject was instructed to fixate. The fixation cross was displayed alone for $750 \mathrm{~ms}$, followed by the first frame with the fixation cross for $500 \mathrm{~ms}$, immediately followed by the second frame and cross for $500 \mathrm{~ms}$. Two icons showing the CW and CCW percepts (similar to Figures $1 \mathrm{~b}, \mathrm{c}$ ) were then displayed on the right and left sides of the window (chosen randomly), each at a distance of 200 pixels from the window's horizontal center. The subject selected and clicked on icon corresponding to his or her perceived motion, using the computer mouse.

\subsubsection{Procedure}

The main experimental block consisted of 48 trials, composed of two sub-blocks of 24 trials, in which the stimuli were presented in each of the 24 angles, in random order (different random order for every subject). The order of the stimuli was identical in the two subblocks, in order to measure subjects' consistency. Prior to the main experimental block, subjects were given written instructions, and performed 20 practice trials, similar to the trials in the main block but excluded from data analysis. Trials followed one another without pause, with breaks every 16 trials. The main experimental block had a median duration of $2.6 \mathrm{~min}$. 
(b)

(a)

\section{1}

(2)

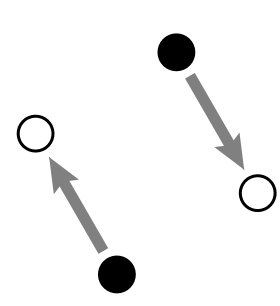

(d)

CW

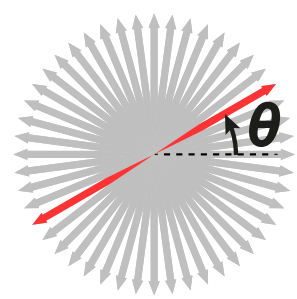

(c)

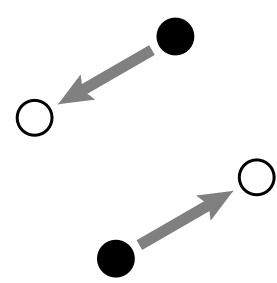

(e)

$\mathrm{CCW}$

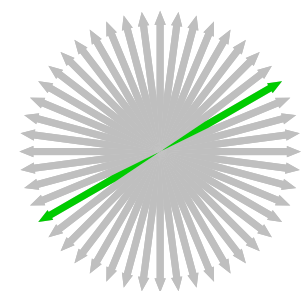

Figure 1: Visual stimuli and representation of responses. (a) The motion quartet stimulus consists of two frames, labeled (1) and (2), each composed of two circles on opposite vertices of an imaginary square. The only parameter that varied from trial to trial was the overall orientation of the figure, angle $\theta$. (b, c) The apparent motion perceived between frames 1 and 2 is ambiguous: it can be seen along one of two axes that differ by $90^{\circ}$. One of the two perceptions happens to be globally clockwise $(\mathrm{CW}, \mathrm{b})$ and the other counterclockwise (CCW, c). (d, e) The subject's task was to indicate perceived motion direction by selecting one of two icons, (b) or (c). The response will be represented as two opposite-facing arrows (because of the $180^{\circ}$ symmetry), colored red if the subject reported CW motion, and green for CCW motion. Each of the gray arrows represents a stimulus orientation that was shown (in random order), so each will be colored red or green in accordance with the response given.

\subsubsection{Subjects}

106 subjects took part in the experiment (40 men and 66 women, ages 18-66, median 27). Prior to taking part in the current experiment, these subjects had participated in a separate internet-based longitudinal study on the evolution of individual biases in the perception of ambiguous visual stimuli (Exp. 3 in Wexler et al., 2015). The stimuli used in the prior longitudinal study were different from those used here. The subjects had been originally recruited through an announcement to a mailing list of several thousand people willing to participate in cognitive science experiments (organized by the Relais d'information sur les sciences de la cognition (RISC), Paris, France). The current experiment was carried out as the final session of the prior longitudinal study. Subjects were paid $1 €$ for their participation in this session. Informed consent was obtained prior to the experiment in accordance with the Declaration of Helsinki. In France, the legal ethics committees do not examine non-invasive behavioral studies. 

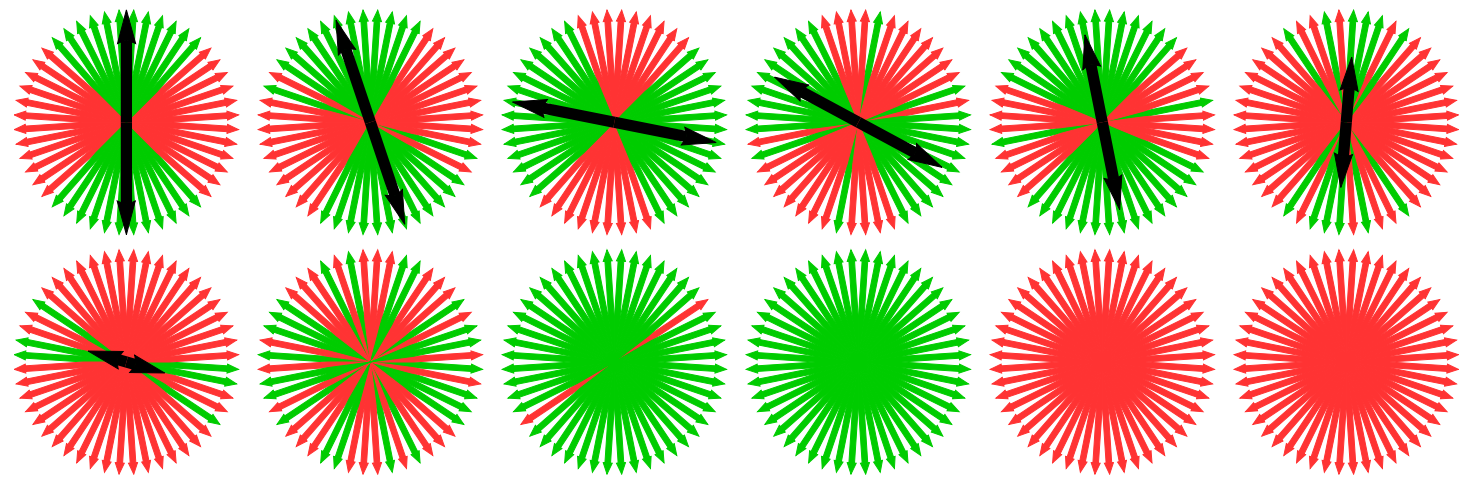

Figure 2: The results of 12 representative subjects in Experiment 1. The data is represented using the graphical representation explained in Figures 1d and e. Each trial is shown as two opposite-facing arrows, corresponding to the orientation of the quartet stimulus. The arrows are colored red if clockwise motion was reported, green if counterclockwise. For clarity, only the data from the first sub-block are shown. Subjects are ordered so that those with axial biases appear in the beginning, and those with rotational biases at the end. For subjects who have a significant axial bias, the preferred axis is shown in black. The full data, of all 106 subjects and both sub-blocks, are shown in Figure A1 in the Appendix.

\subsection{Results}

The results of 12 representative subjects are shown in Figure 2, using the graphical representation explained in Figures $1 \mathrm{~d}$ and e. For clarity, only the data from the first sub-block are shown. The full data, of all 106 subjects and both sub-blocks, are shown in Figure A1 in the Appendix.

A simple hypothesis is that the perceptual decision on each trial is a stochastic choice, with $50 \%$ chance of responding $\mathrm{CW}$ and $50 \% \mathrm{CCW}$, independent of all other trials. If so, the response patterns should resemble random, isotropic mixtures of red and green arrows (because stimulus orientations were presented in random order). This is visibly not the case for a majority of subjects (as can be seen in Figures 2 and A1), whose data show large-scale spatial patterns.

The randomness of the response patterns was tested using a procedure based on the number of boundaries between $\mathrm{CW}$ and CCW responses. If one considers $n=48$ independent binary variables on a circle (with equal probability of $\mathrm{CW}$ and $\mathrm{CCW}$ values), the number of boundaries $b$ between $\mathrm{CW}$ and CCW regions follows a binomial distribution $P(b, n)=n ! /\left[2^{n-1} b !(n-b) !\right]$, which was taken as the null hypothesis. The large-scale spatial structure of many of the response patterns, with large homogeneous regions, results in an improbably small number of boundaries, as in the Wald-Wolfowitz runs test (1940). The Benjamini-Hochberg correction (1995) was applied to take into account multiple tests, with false-discovery ratio set to 0.05 . Using this procedure, I found that $71 \%$ of the subjects had response patterns that differed significantly from random and indepen- 

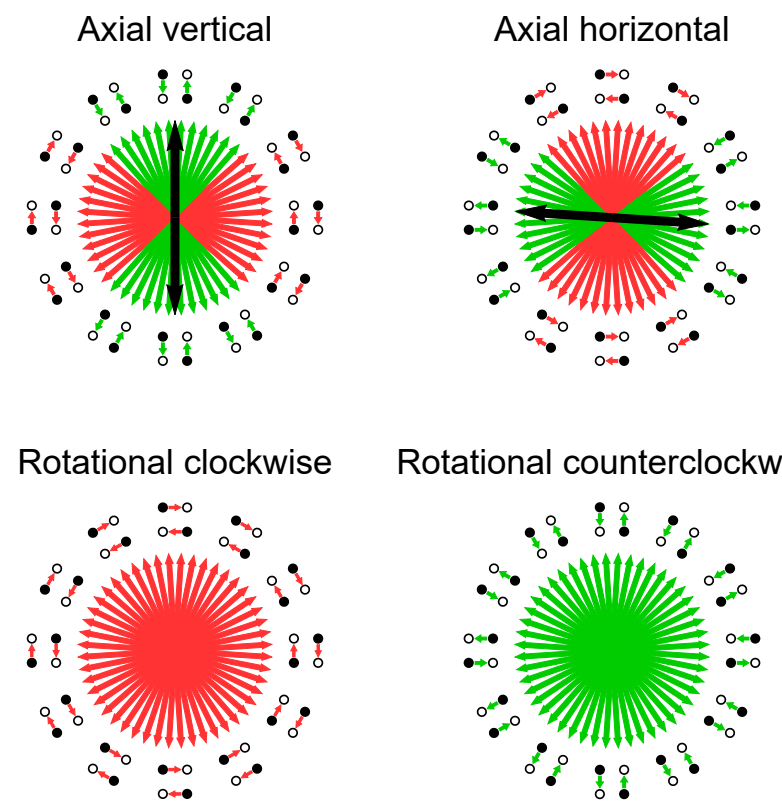

Figure 3: Four main bias patterns, illustrated by data of four subjects in Experiment 1. The data is presented in the same way as previously: each stimulus orientation is represented by two opposite-facing arrows, colored red if the subject reported CW motion, and green for $\mathrm{CCW}$ motion. The icons at the edges show the subjects' percepts at corresponding stimulus orientations. The top patterns illustrate axial biases, with nearly vertical and horizontal preferred axes. The bottom patterns show rotational biases, with clockwise and counterclockwise preferred rotation directions.

dent choices of $\mathrm{CW}$ or $\mathrm{CCW}$ at each orientation.

In fact, as can be seen in Figures 2 and A1, most subjects' response patterns seem to cluster around two distinct types. In one pattern, many subjects' responses show four alternating CW/CCW regions of about equal size (i.e., $90^{\circ}$ ). Subjects presenting this pattern are shown at the beginning of Figures 2 and A1. This pattern is indicative of an axial bias, or a preference for translation, in either direction, closest to a particular axis. This is illustrated in Figure 3. The overall orientation of the four-region pattern determines the preferred axis, as shown in the top line of Figure 3.

A second, qualitatively different pattern can be seen in subjects at the end of Figures 2 and A1: all or nearly all responses being either CW or CCW. This kind of pattern will be called a rotational bias, a preference for motion compatible with either clockwise or counterclockwise rotation.

When individual data was tested for the two bias patterns, sizable fractions of the subject pool showed statistically significant biases of one or the other type: $41 \%$ of the subjects had a significant axial bias, while $45 \%$ had a significant rotational bias (with corrections for multiple tests using a Benjamini-Hochberg procedure with a false-discovery rate of 0.05 ). The axial bias was tested as follows: for each trial, I took one of the two re- 
(a)

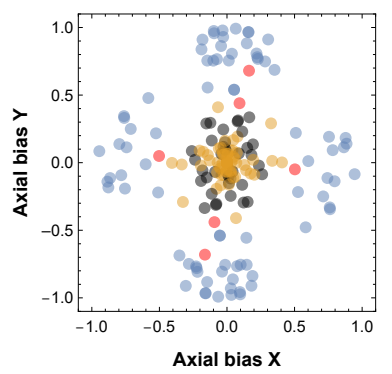

(b)

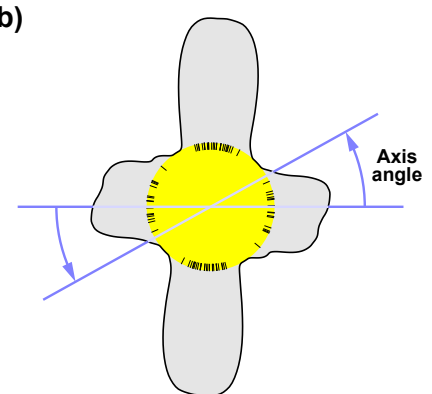

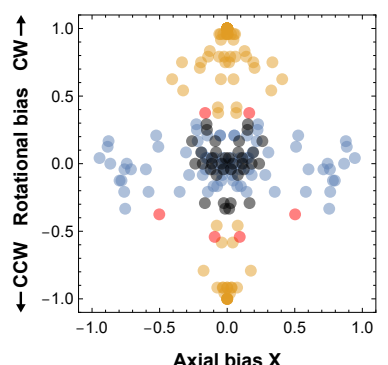

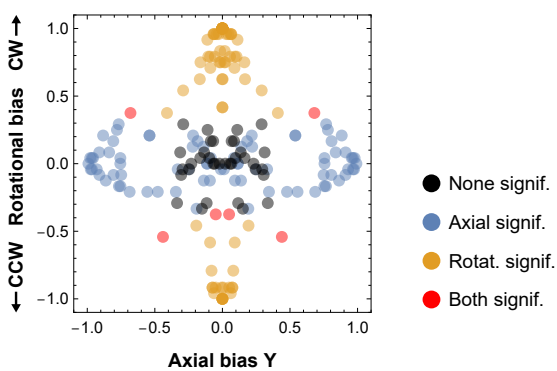

(c)

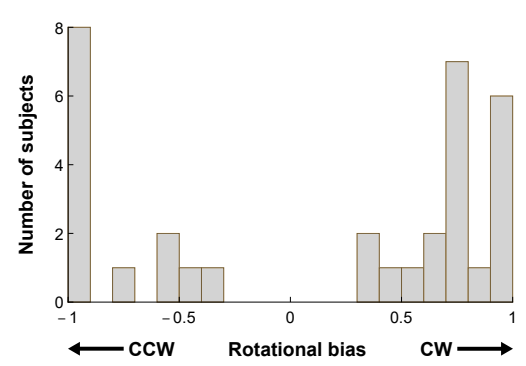

Figure 4: Individual biases in Experiment 1. (a) A 3D representation of the biases. Each point represents one subject. The first two dimensions are the $x$ and $y$ components of the axial bias, and the third dimension the rotational bias. The color of the points reflects the results of statistical tests of the two bias types. (b) The distribution of axis directions for subjects with a statistically significant axial bias. Each subject's axis is represented by two marks on opposite sides of the yellow circle. The curve is the probability density function of axis directions, obtained by smoothing the directions using a semicircular kernel with width $15^{\circ}$. (c) The distribution of rotational biases for subjects with significant rotational bias. This quantity runs from -1 (corresponding to all responses CCW) to +1 (all CW).

ported translation directions, and multiplied the angle by 2 (the standard way of handling axial data), and applied the Rayleigh test Mardia \& Jupp, 1999. The orientation of the preferred axis (the black arrows in Figures 2 and A1 was calculated by multiplying the angle of the perceived axis vector by 2 , taking a circular mean, and diving the resulting angle by 2 . Rotational bias was tested using a sign test on the raw response data.

Individual biases are represented in Figure $\underline{4}$ as points in a 3D space. The first two dimensions are the cartesian components of the preferred axis vector, whose direction is the orientation of the preferred translation axis, and whose length is the strength of the axial bias. (Since axial bias is bi-directional, each subject is represented by two opposite points in the first two dimensions.) The third dimension is the rotational bias, calculated by taking the mean of each subject's raw responses, with +1 corresponding to $\mathrm{CW}$ and -1 to CCW. The subjects with a significant axial bias are colored blue in Figure $4 \mathrm{a}$, and those having a significant rotational bias colored yellow. 
Interestingly, there were 3 subjects who had statistically significant biases of both the axial and the rotational types (colored red in Figure 4). This result is important because it shows that the two bias types are not mutually exclusive. Rather, individual biases are properly seen as a multidimensional combinations of the two underlying bias types. Although only a small fraction of subjects had significant axial and rotational biases, many more appear to have combinations of the two bias types (Figure A1), and would have likely been significant in an experiment with a larger volume of data (more than 48 trials).

In the subjects with significantly axial patterns, the distribution of axial directions was highly non-uniform (see Figure 4b), with peaks in the cardinal directions (88\% of the subjects had axis directions closer to the cardinals than to the main diagonals, significantly above $50 \%$ by bootstrap), and with a higher peak in the vertical than in the horizontal direction ( $63 \%$ of the axes closer to vertical than horizontal, significantly above $50 \%$ by bootstrap). Of the subjects with a significant rotational pattern of responses, a majority ( $73 \%$, significantly above $50 \%$ by bootstrap) had a bias in the CW direction (Figure 4c).

\subsection{Discussion}

The results of this experiment have shown that when the motion quartet is presented at different orientations on successive trials, perceptual decisions about the direction of motion depend on large-scale bias patterns. The bias patterns seem to be multi-dimensional combinations of two extreme types. The first type is axial bias, favoring translation, in either direction, along a given axis (which can vary between $0^{\circ}$ and $180^{\circ}$ ). The second type of bias pattern is rotational, favoring motion that is globally clockwise or counterclockwise. Quite a few subjects have bias patterns that are pure or almost pure axial, or rotational. Other subjects have patterns that seem like combinations of axial and rotational, with several subjects having statistically significant biases of both types.

If a subject has an axial bias with a vertical axis, or an axial bias with a horizontal axis, or a clockwise rotational bias, or a counterclockwise one, or some combination of axial and rotational biases-is this a permanent condition? In our previous work on individual perceptual biases, it has been shown that such biases are on the whole stable, but also sometimes change, either continuously or discontinuously (Wexler et al., 2015). In our previous study, we probed the temporal evolution of the individual biases with daily measurements over a period of several months. In the second experiment in the current study, I measured changes over time in the bias pattern for the motion quartet, testing each subject 45 times over a period of 9 hours.

\section{Experiment 2: Temporal evolution of individual biases}

In this experiment I directly tested the stability of the individual bias patterns, by measuring them every 12 minutes over a period of 9 hours, for a total of 45 measurements in every subject. One possibility is that the bias patterns are stable, at least over this 
duration. A second possibility is that the bias type-axial or rotational-remains stable, but with the axis or direction of rotation changing over time. Finally, it is possible that biases vary over their parameter space (such as Figure $4 \mathrm{a}$ ), which would additionally allow changes from axial to rotational biases, or vice versa.

There is a possible criticism of the conclusion from Experiment 1 that the individual differences in the bias patterns are somehow intrinsic to the subject: the variety of individual response patterns (e.g., Figure 2) could have been due to the different order of trials for every subject. For example, perhaps the first few orientations seen by each subjects somehow fixed the subject's subsequent response pattern. If so, one should find much less interindividual variation if the trials were in the same order for all subjects. This was tested in Experiment 2, in which trials were always in one and the same (but 'random') order for all subjects, and for all blocks for every subject.

\subsection{Methods}

In contrast to Experiment 1, this experiment was performed in the laboratory.

The stimulus was very similar to that in Experiment 1. On every block a single repetition of the same 24 orientations as in Experiment 1 was tested. The orientations were presented in random order, but in the same random order on every block, for every subject. (This was done in order to test whether the variety of individual response patterns found in Experiment 1 could have been due to the different order of trials for every subject.)

Stimulus disks were presented with their centers at $2^{\circ}$ of visual angle from the center of the monitor. Each disk had radius of $0.25^{\circ}$ of visual angle, and was white $\left(112 \mathrm{~cd} / \mathrm{m}^{2}\right)$ on a black background $\left(0.85 \mathrm{~cd} / \mathrm{m}^{2}\right)$. Subjects were instructed to fixate a red disk drawn at the center of the monitor (radius $0.1^{\circ}$ of visual angle). On each trials the fixation disk was presented alone for $500 \mathrm{~ms}$, followed each of the two frames (with the fixation disk) presented for $400 \mathrm{~ms}$, followed by the response icons, as in Experiment 1. Stimuli were presented on a Sony GDM F520 monitor (resolution $1024 \times 768$, refresh rate $120 \mathrm{~Hz}$, size approximately $38 \times 29^{\circ} \mathrm{dva}$ ) at an approximate distance of 57.3 from the subject, whose head was restrained with a chinrest during the experimental blocks. The experiment was performed in a room with normal indoor lighting.

Blocks began every 12 minutes. The median duration of one block was about $54 \mathrm{~s}$, so subjects had a break of about 11 minutes between blocks. During these breaks they were free to do what they wished, for example to read, study, listen to music, eat, and so on; they could also leave the experimental room, for example to use the restroom or smoke. $30 \mathrm{~s}$ before the start of each block, the computer emitted a series of beeps and flashes to warn the subject to put his or her head into the chinrest, and that the block was about to begin. Before the start of the series of 45 blocks, subjects performed a practice block, identical to the experimental blocks. Six subjects took part in the experiment (ages 23 to 37 , median 28 , 4 women and 2 men), and were paid $10 € /$ hour. 


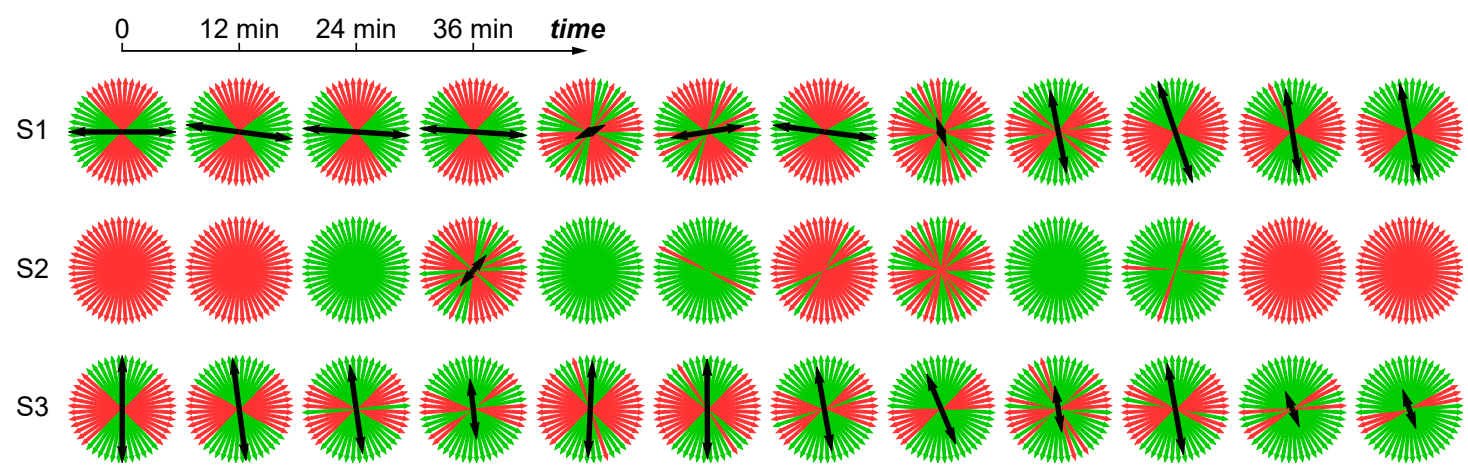

Figure 5: Examples of time evolution of the bias patterns in three subjects in Experiment 2. Each row represents 12 measurements in a single subject, made at 12-minute intervals, over a period of about 2.2 hours.

\subsection{Results}

The complete time series of the bias patterns of all six subjects are shown in Figure A2, in the Appendix. The first thing to note about these data is that, clearly, the samples are not independent of one another; in most cases, the shape of the bias pattern is retained from one measurement to the next. To check this, I performed a test based on the fact that the number of differences between two independent binary samples follows the binomial distribution. The number of differences in successive samples was calculated, and the Benjamini-Hochberg procedure (false discovery rate 0.05 ) was used to correct for multiple tests. In $79 \%$ of the cases, responses in successive blocks differed by significantly fewer trials than predicted by the null (no-memory) hypothesis. Thus, one can conclude that bias patterns are generally stable from one measurement to the next, 12 minutes later.

Nevertheless, there are changes in the bias patterns over time. Three examples of change, excerpted from the time series of three separate subjects (12 blocks, about 2.2 hours), are shown in Figure 5 . In the first series in Figure 5 a horizontal axial bias becomes roughly vertical, while in the second series a rotational bias cycles between $\mathrm{CW}$ and CCW several times. In the third series a strong axial bias undergoes a transition to an almost completely rotational bias, while seemingly retaining a small 'memory' of its previous axial direction. (This memory could be the result of taking the shortest path between the two biases in the 3D space of Figure 4a.) Thus, the bias patterns seem to be able to change within type-change of preferred axis in axial bias, change of preferred rotation direction in rotational bias-as well as between types-change from axial to (mostly) rotational bias.

The behavior of the time series was analyzed in a different way, by calculating mean response (with individual responses coded as $+1=\mathrm{CW},-1=\mathrm{CCW}$ ) for each session, and analyzing the time series of the means using the autoregressive integrated movingaverage (ARIMA) framework (Box \& Jenkins, 2008). The analysis, using the forecast package in $\mathrm{R}$ (Hyndman \& Khandakar, 2008), automatically identified the best model by searching through $\operatorname{ARIMA}(p, d, q)$ models with $p, d, q \in\{0,1\}$. The search was performed 
by applying the KPSS unit-root test (Kwiatkowski et al., 1992) to determine the differencing order $(d)$, and then searching the $p, q$ subspace for the model that best fits the series, subject to penalties from the Akaike information criterion (Hyndman \& Khandakar, 2008). The same analysis was carried out on the 3-month time series by Wexler et al. (2015).

The most common time series model found was $\operatorname{ARIMA}(0,1,1)$, identified by the automatic method for three subjects as the best model. This model is equivalent to an underlying random-walk or Brownian-noise variable, observed through independent measurement noise (Box \& Jenkins, 2008; Wexler et al., 2015). The time series of two other subjects were fitted by $\operatorname{ARIMA}(1,1,0)$ and ARIMA $(1,0,0)$ models, while the final subjectthe same one who had no significant autocorrelations-was fitted with a white-noise $\operatorname{ARIMA}(0,0,0)$ model.

\subsection{Discussion}

By repeatedly measuring bias patterns to obtain time series of significant length, the results of this experiment showed that the bias patterns at successive samples (separated by 12 minutes) were not independent, but generally remain stable over time. However, there were significant changes, both within bias types (changes in preferred axis for axial biases, and in preferred rotation direction for rotational biases), and between bias types (an axial bias that became a nearly complete rotational bias).

A time-series analysis of mean responses, averaged over stimulus orientation, revealed that the time series of a plurality of our subjects were best described by one particular model, a random walk measured through noise. The same result was obtained by Wexler et al. (2015) on different measures coming from biases pertaining to different stimuli (biases for surface orientation in structure-from-motion stimuli, and biases for motion direction in transparency-from-motion stimuli). This model, as well as the other time-series results, fit in with a picture that the individual biases are reflections (through noisy measurements) of persistent brain states. These brain states evolve, either through exposure to external stimuli or through internal neural dynamics, and their evolution can at least partly be described as a random walk.

It should be noted that, although in this experiment trials were always in the same order for all subjects and all blocks (in contrast to Experiment 1), there was wide variability, both between and within subjects (Figure A2). Thus, variation in response patterns is due to variable and evolving states of the visual system, rather than different order of stimuli in Experiment 1. Nor could have the between-subject variability in Experiment 1 been (all) due to differences in experimental conditions in that internet-based experiment, as the data in Experiment 2-performed in controlled laboratory conditions-also shows a great deal of between-subject variability. 


\section{Experiment 3: Involuntary perception or voluntary con- trol?}

So far we have implicitly interpreted the differences in bias patterns-both between subjects (Experiments 1 and 2) and over time within the same subject (Experiment 2)—as involuntary perceptual effects due to internal state changes in the visual system. However, some observers can sometimes voluntarily control their percepts. Although subjects certainly weren't instructed to voluntarily control their perceptions, and had no obvious reason to do so (except possibly boredom in Experiment 2), so far we cannot exclude that bias differences in Experiments 1 and 2 were at least partly due to voluntary, decisional factors rather than involuntary, perceptual ones.

Voluntary control of the direction of motion in the motion quartet was noticed by Ramachandran and Anstis (1983b; 1985), who remarked that their continuously cycling quartet display was controllable voluntarily for temporal frequencies below roughly $3 \mathrm{~Hz}$. In our single-shot (two-frame) version of the quartet, we have noticed that the critical factor is, in fact, the duration of the first frame. We have found that when this duration is above roughly $200-400 \mathrm{~ms}$, some observers can reliably voluntarily control the perceived direction of motion. When the duration of the first frame is below about $200 \mathrm{~ms}$, we have found no observer who can control the direction of motion. These observations are at least in qualitative agreement with those of Ramachandran and Anstis (1983b; 1985). The reader is invited to try to voluntarily control the motion quartet at various frame durations using an on-line demo: http://lab-perception.org/demo/qvol.

The goal of the present experiment was to repeat the time series measurements of idiosyncratic biases of Experiment 2, but in conditions in which voluntary control would have been impossible. In practice, I searched for a duration threshold for the first frame below which subjects could not voluntarily control the perceived direction of motion. To measure this threshold, I performed an auxiliary experiment, described the Supplementary Materials Section A1 because it is peripheral to the main topic of this article. In this experiment subjects were instructed, for first-frame durations from 200 to $1000 \mathrm{~ms}$, to try to force themselves to perceive either vertical, horizontal, clockwise, or counterclockwise motion-but were nevertheless told to truthfully report which of the usual two motions they perceived on any given trial. Of course subjects could have simply reported having seen what they were asked to force themselves to see. However, this would have only exaggerated the measured effectiveness of voluntary control, or in other words decreased the temporal threshold of voluntary control, which is not a problem for the present purpose. It was found that the degree of voluntary control increased significantly as a function of first-frame duration. There was no measurable control at $200 \mathrm{~ms}$. For the reason stated above, this represents a lower bound for the control threshold. The reader is referred to Section $\underline{\mathrm{A} 1}$ for further details.

Because the durations of the first frame in Experiments 1 and 2 were 500 and 400 $\mathrm{ms}$, respectively, it cannot be excluded that subjects had some voluntary control of the perceived motion direction. I therefore performed another experiment, similar to Experiment 2, but with the duration of the first frame set to $100 \mathrm{~ms}$, well below the lower 


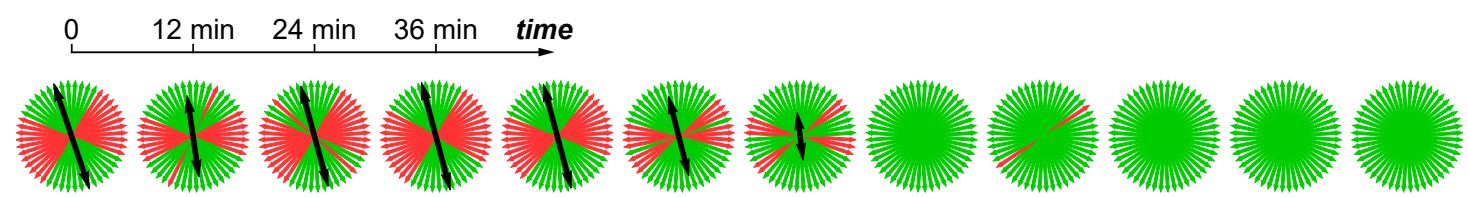

Figure 6: Example of time evolution of the bias pattern in one subjects in Experiment 3 . The graphs represent 12 measurements made at 12 -minute intervals, over a period of about 2.2 hours.

threshold for voluntary control. In all other respects, the conditions were identical to those of Experiment 2. Six naive subjects, none of whom had participated in any other experiment in this study, took part in this experiment.

\subsection{Results}

The time series for all subjects are shown in Figure A3 in the Appendix. The bias patterns are perhaps a bit noisier than in Experiment 2 (Figure A3), but the general features found in time series of Experiment 2 are present. The memory effect is still found: patterns on successive measurements tend to be alike. Quantitatively, calculating the number of different responses in successive samples, in $69 \%$ of the cases, responses in successive blocks differed by significantly fewer trials than predicted by the null, no-memory hypothesis.

Significantly, there were cases of bias change over time. Namely, one subject with a vertical bias pattern several times either lost a clear bias (second subject in Figure A3), or developed a counterclockwise bias, each time for several blocks and each time eventually regaining vertical bias (3rd subject in Figure A3). Another subject, part of whose time series is shown in Figure 6 , who initially had a very strong axial bias, with an approximately vertical axis, gradually underwent a change of bias to a very strong counterclockwise bias.

It is worth noting two things about the time series of bias patterns in Figure $\underline{6}$. First, during the axial phase the preferred axis is not exactly vertical, but slightly counterclockwise of vertical. What is interesting is that this deviation from vertical seems to be systematic: it actually holds for 17 blocks (over 3 hours) before the axial bias disappears (as can be seen at the bottom of Figure A3). Thus, this counterclockwise deviation is most likely $\left(p=2^{-(17-1)} \approx 1.5 \times 10^{-5}\right.$ ) part of the actual internal state of the visual system, rather than noise. Second, the change from axial to rotational bias is gradual (over about 30 minutes) and monotonic, an orderly path in parameter space (Figure $\underline{4}$ ).

\subsection{Discussion}

This experiment was a replication of Experiment 2, but in conditions where no voluntary control of the perceived direction of motion should be possible. The requisite conditionsa first frame with duration $100 \mathrm{~ms}$ - were determined through an auxiliary experiment, described in Section A1. The results replicated the essential features of Experiment 2, namely idiosyncratic bias conditions that are usually stable over time, but that occasion- 
ally undergo changes that are sometimes large. This shows that these effects are not due, or at least not solely due, to conscious, volitional effects, but are truly perceptual in nature.

\section{General discussion}

The results of this study show that complex structures underlie the perception of the motion quartet. When a a two-frame quartet is shown at orientations that vary from trial to trial, responses as a function of orientation are organized around two distinctive types of pattern in most subjects. One of the patterns consists of four clockwise/counterclockwise lobes of about $90^{\circ}$, which corresponds to a bias for translation (in either direction) along a preferred axis, whose orientation depends on the orientation of the pattern. In subjects who have this pattern the distribution of preferred axes has peaks in the cardinal directions, with the vertical direction more common than the horizontal. The other pattern is a prevalence of uniformly clockwise or counterclockwise responses, a bias for rotation in one of these directions. In subjects with rotational bias, clockwise preference is more common than counterclockwise.

The two bias patterns are actually extremes in a continuum. Although many subjects have the extreme patterns, others have combinations of axial and rotational biases. Thus, individual biases patterns are actually points in a multidimensional space, with several subjects having biases that have significant contributions of both bias types.

A second experiment, using repeated measurements over nine hours, showed that biases are usually stable over time, but also sometimes undergo significant change. I have found cases where an axial bias changes preferred axis, and a rotational bias changes preferred direction. In at least one case, an axial bias became almost completely rotational.

Finally, two control experiments showed that the variety of bias patterns found across subjects, and the patterns' changes over time within subjects, are not due to voluntary or decisional processes. The first control experiment revealed that voluntary control of the motion quartet is possible only when the duration of the first frame is above a threshold of 200-400 ms. Since the duration of the first frame in Experiments 1 and 2 was slightly above this threshold, I performed another control experiment, similar to the time series measurement of Experiment 2, but with the duration of the first frame set to $100 \mathrm{~ms}$, well below the threshold for voluntary control. Results similar to those of Experiment 2 show that between- and within-subject variability cannot be due to voluntary or decisional effects.

Certain findings presented here agree with earlier observations on the motion quartet, but place these earlier observations in a broader context. It has long been known that, when the quartet is in its usual upright orientation, perception of vertical motion is more common than that of horizontal motion (Gengerelli, 1948), with this difference attributed to the difference between intra- and inter-hemispheric connections (Genç et al., 2011; Shimono et al., 2012). This discrete fact can now be seen as part of a larger picture: observers who have an axial bias have a preferred axis with a population distribution estimated by Figure $4 \mathrm{~b}$. This distribution has a larger peak centered on vertical than 
horizontal orientations, but, interestingly, oblique preferred axes also occur.

However, it is important to note that the temporal changes in preferred axis observed in Experiment 2, as well as changes from axial to rotational bias, invalidate a strong version of the hypothesis that brain anatomy alone determines individual differences in bias (Genç et al., 2011; Shimono et al., 2012). However, brain anatomy may play a role in the vertical-horizontal asymmetry in the population distribution of preferred axes.

Another observation that enlarges previously known facts is that, when the quartet is shown over several cycles, some observers perceive a rotation in a consistent direction (clockwise or counterclockwise), rather that back-and-forth translation along a particular axis (Ramachandran \& Anstis, 1983b). Once again, this can be seen in the context of the current findings, as likely to arise in subjects having rotational (rather than axial) bias. Interestingly, the consistent rotation percept is reported as disappearing above a certain temporal frequency (Ramachandran \& Anstis, 1983b), which may indicate that individual bias patterns depend on temporal frequency-a possibility not tested here.

Adding to our previous results on individual biases (Wexler et al., 2015), the current study demonstrates an additional family of stimuli governed by robust-but-evolving biases with large individual differences. However, the biases governing the motion quartet are more complex than the SFM and TFM biases reported by Wexler et al. (2015), which were shown to exist in a two-dimensional parameter space (i.e., a large part of betweenand within-subject variability can be described by two parameters). Here I have shown that the motion quartet biases exist in a three-dimensional space, but their actual dimensionality may be even higher. For example, if it is found the bias patterns depend idiosyncratically on temporal frequency (as is likely-see previous paragraph), position in the visual field, stimulus size, or other stimulus parameters, the dimensionality of the biases will be shown to be higher than three. Other studies of idiosyncratic biases may have also demonstrated high-dimensional biases (Carter \& Cavanagh, 2007; Afraz et al., 2010; Houlsby et al., 2013; Kosovicheva \& Whitney, 2017), but precise estimation of dimension is difficult. The question is important because the biases are actually brain states that can be measured psychophysically, that govern perception of families of stimuli, and that evolve in response to stimuli or autonomously. Understanding the spaces in which they evolve and the laws governing this evolution is crucial to complete description of perception.

\section{Acknowledgments}

This work was partly supported by the ERC PATCH grant. I am grateful to Arielle Veenemans and Hamdi Habacha for collecting the time series data.

\section{References}

Afraz, A., Pashkam, M. V., \& Cavanagh, P. (2010). Spatial heterogeneity in the perception of face and form attributes. Current Biology, 20(23), 2112-2116. 
Anstis, S. \& Ramachandran, V. S. (1987). Visual inertia in apparent motion. Vision Research, 27(5), 755-764.

Attneave, F. (1971). Multistability in perception. Scientific American, 225(6), 63-71.

Benjamini, Y. \& Hochberg, Y. (1995). Controlling the false discovery rate: a practical and powerful approach to multiple testing. Journal of the Royal Statistical Society. Series $B$ (Methodological), 289-300.

Bessel, F. W. (1823). Astronomische Beobachtungen auf der Königlichen Universitäts Sternwarte in Königsberg. VIII Abtheilung. Königsberg: Universitäts-Buchhandlung.

Blake, R. \& Logothetis, N. K. (2002). Visual competition. Nature Reviews. Neuroscience, $3(1), 13-21$.

Box, G. E. P. \& Jenkins, G. M. (2008). Time Series Analysis: Forecasting and Control (4 edition). Hoboken, N.J: Wiley.

Carter, O. \& Cavanagh, P. (2007). Onset rivalry: brief presentation isolates an early independent phase of perceptual competition. PloS One, 2(4), e343.

Carter, O., Konkle, T., Wang, Q., Hayward, V., \& Moore, C. (2008). Tactile rivalry demonstrated with an ambiguous apparent-motion quartet. Current Biology, 18(14), 10501054.

Coren, S. \& Porac, C. (1987). Individual differences in visual-geometric illusions: predictions from measures of spatial cognitive abilities. Perception \& Psychophysics, 41(3), 211-219.

Genç, E., Bergmann, J., Singer, W., \& Kohler, A. (2011). Interhemispheric connections shape subjective experience of bistable motion. Current Biology, 21(17), 1494-1499.

Gengerelli, J. (1948). Apparent movement in relation to homonymous and heteronymous stimulation of the cerebral hemispheres. Journal of Experimental Psychology, 38, 592-599.

Goutcher, R. (2016). Motion direction influences surface segmentation in stereo transparency. Journal of Vision, 16(15), 17.

Grzeczkowski, L., Clarke, A. M., Francis, G., Mast, F. W., \& Herzog, M. H. (2017). About individual differences in vision. Vision Research. In press.

Haladjian, H., Anstis, S. M., Seizova-Cajic, T., Wexler, M., \& Cavanagh, P. (n.d.). The tactile quartet: Comparing ambiguous apparent motion in tactile and visual stimuli.

Hock, H. S., Kelso, J. A., \& Schöner, G. (1993). Bistability and hysteresis in the organization of apparent motion patterns. Journal of Experimental Psychology. Human Perception and Performance, 19(1), 63-80.

Houlsby, N. M. T., Huszár, F., Ghassemi, M. M., Orbán, G., Wolpert, D. M., \& Lengyel, M. (2013). Cognitive tomography reveals complex, task-independent mental representations. Current Biology, 23(21), 2169-2175.

Hyndman, R. J. \& Khandakar, Y. (2008). Automatic time series forecasting: The forecast package for R. Journal of Statistical Software, 27(3).

Kleinschmidt, A., Sterzer, P., \& Rees, G. (2012). Variability of perceptual multistability: from brain state to individual trait. Philosophical Transactions of the Royal Society B: Biological Sciences, 367(1591), 988-1000. 
Kosovicheva, A. \& Whitney, D. (2017). Stable individual signatures in object localization. Current Biology, 27(14), R700-R701.

Kwiatkowski, D., Phillips, P. C. B., Schmidt, P., \& Shin, Y. (1992). Testing the null hypothesis of stationarity against the alternative of a unit root: How sure are we that economic time series have a unit root? Journal of Econometrics, 54(1-3), 159-178.

Leopold, D. A. \& Logothetis, N. K. (1999). Multistable phenomena: changing views in perception. Trends in Cognitive Sciences, 3(7), 254-264.

Liaci, E., Bach, M., Tebartz van Elst, L., Heinrich, S. P., \& Kornmeier, J. (2016). Ambiguity in Tactile Apparent Motion Perception. PloS One, 11(5), e0152736.

Locke, J. (1690). An essay concerning human understanding. London: Bassett.

Long, G. M. \& Toppino, T. C. (2004). Enduring interest in perceptual ambiguity: alternating views of reversible figures. Psychological Bulletin, 130(5), 748-768.

Maloney, L. T., Martello, M. F. D., Sahm, C., \& Spillmann, L. (2005). Past trials influence perception of ambiguous motion quartets through pattern completion. Proceedings of the National Academy of Sciences of the United States of America, 102(8), 31643169 .

Mamassian, P. \& Wallace, J. M. (2010). Sustained directional biases in motion transparency. Journal of Vision, 10(13), 23.

Mardia, K. V. \& Jupp, P. E. (1999). Directional Statistics. Chichester: Wiley.

Necker, L. A. (1832). Observations on some remarkable optical phænomena seen in Switzerland; and on an optical phænomenon which occurs on viewing a figure of a crystal or geometrical solid. Philosophical Magazine Series 3, 1(5), 329-337.

Ramachandran, V. S. \& Anstis, S. M. (1983a). Extrapolation of motion path in human visual perception. Vision Research, 23(1), 83-85.

Ramachandran, V. S. \& Anstis, S. M. (1983b). Perceptual organization in moving patterns. Nature, 304(5926), 529-531.

Ramachandran, V. S. \& Anstis, S. M. (1985). Perceptual organization in multistable apparent motion. Perception, 14(2), $135-143$.

Rubin, E. (1921). Visuell wahrgenommene Figuren. Copenhagen: Gyldendalske Boghandel.

Schiller, P. v. (1933). Stroboskopische Alternativversuche. Psychologische Forschung, 17, 179-214.

Schütz, A. C. (2014). Inter-individual differences in preferred directions of perceptual and motor decisions. Journal of Vision, 14(12), 16.1-17.

Schütz, A. C. \& Mamassian, P. (2016). Early, local motion signals generate directional preferences in depth ordering of transparent motion. Journal of Vision, 16(10), 24.

Shimono, M., Mano, H., \& Niki, K. (2012). The brain structural hub of interhemispheric information integration for visual motion perception. Cerebral Cortex, 22(2), 337344.

Sterzer, P. \& Kleinschmidt, A. (2007). A neural basis for inference in perceptual ambiguity. Proceedings of the National Academy of Sciences of the United States of America, 104(1), 323-328. 
Sterzer, P., Kleinschmidt, A., \& Rees, G. (2009). The neural bases of multistable perception. Trends in Cognitive Sciences, 13(7), 310-318.

Wald, L. \& Wolfowitz, J. (1940). On a test whether two samples are from the same population. The Annals of Mathematical Statistics, 11(2), 147-162.

Wallach, H. (1935). Über visuell wahrgenommene Bewegungsrichtung. Psychologische Forschung, 20, 325-380.

Wexler, M., Duyck, M., \& Mamassian, P. (2015). Persistent states in vision break universality and time invariance. Proceedings of the National Academy of Sciences of the United States of America, 112(48), 14990-14995.

Wheatstone, C. (1838). On some remarkable, and hitherto unobserved, phenomena of binocular vision. Philosophical Transactions of the Royal Society of London, 128, 371-394.

Wilmer, J. B. (2008). How to use individual differences to isolate functional organization, biology, and utility of visual functions; with illustrative proposals for stereopsis. Spatial Vision, 21(6), 561-579.

Witzel, C., O'Regan, J. K., \& Hansmann-Roth, S. (2017). The dress and individual differences in the perception of surface properties. Vision Research. In press.

Zhang, Q.-f., Wen, Y., Zhang, D., She, L., Wu, J.-y., Dan, Y., \& Poo, M.-m. (2012). Priming with real motion biases visual cortical response to bistable apparent motion. Proceedings of the National Academy of Sciences of the United States of America, 109(50), 20691-20696. 
Appendix 


\section{1}
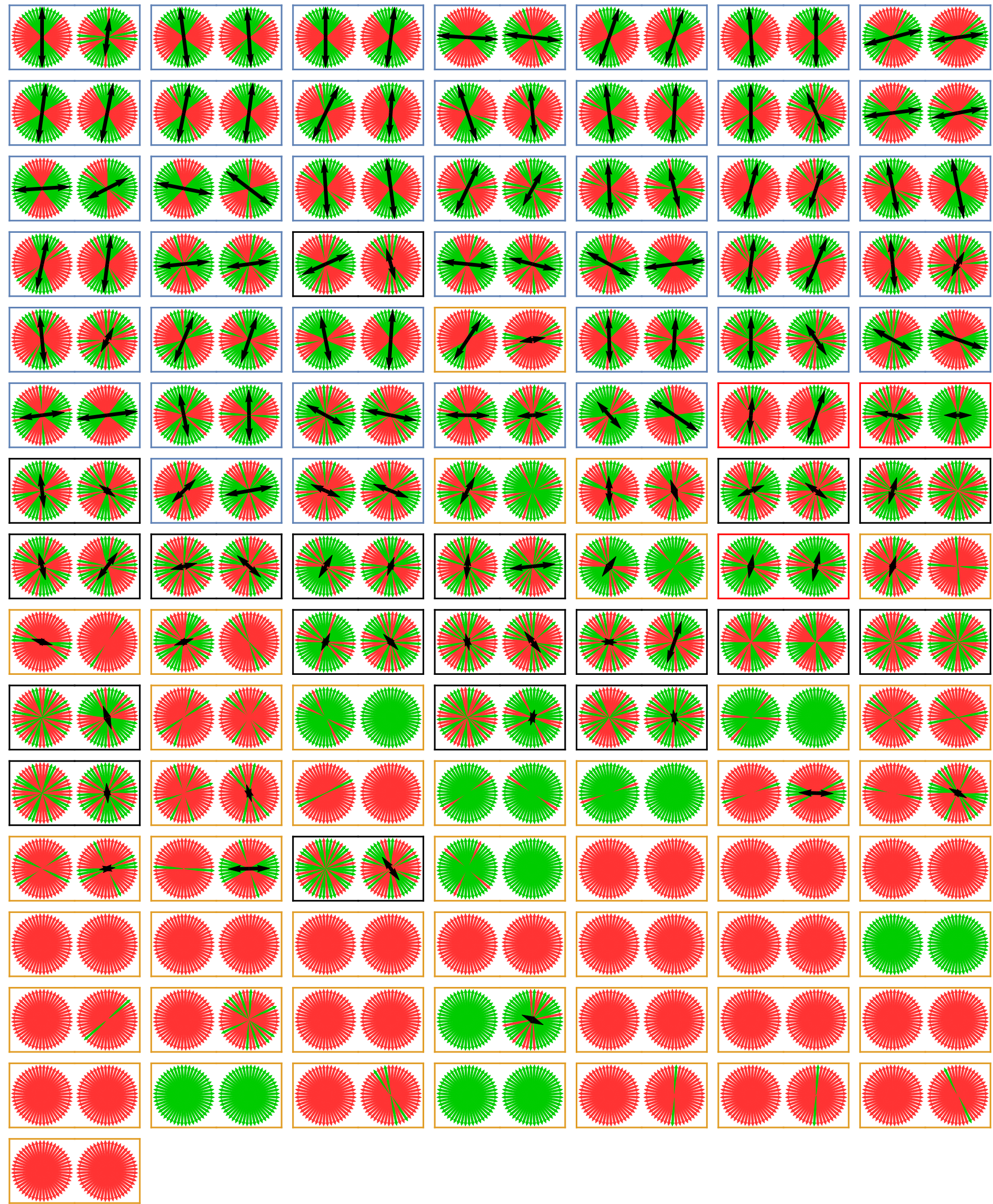

Figure A1: Data of all 106 subjects in Experiment 1, with each rectangle corresponding to one subject. Data from each of the two identical sub-sessions are shown separately. The border color of the rectangle reflects the results of statistical tests of the two bias types (blue: significant axial bias, orange: significant rotational bias, red: significant axial and rotational biases, black: neither significant). Subjects are ordered so that those with axial biases appear in the beginning, and those with rotational biases at the end. 


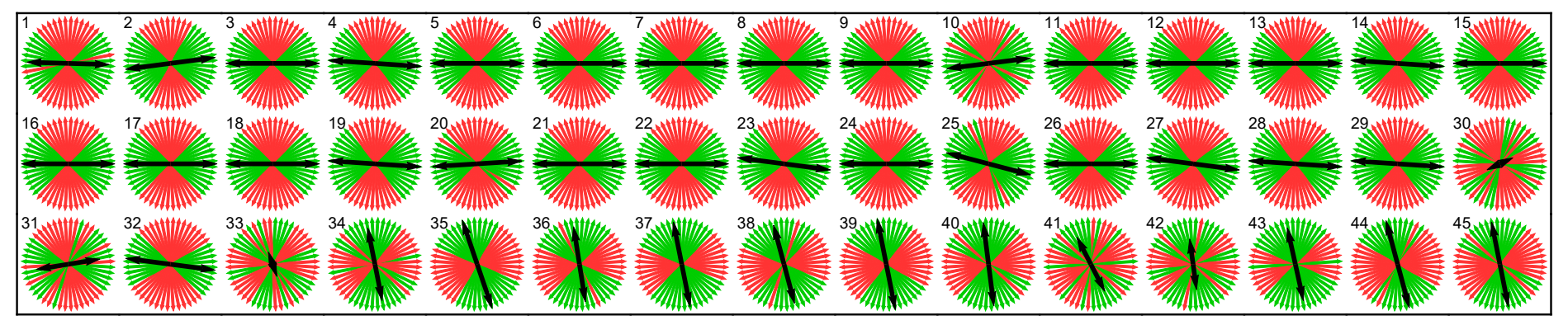

\begin{tabular}{|lllllllllllllllllll}
\hline & 1 & 1 & 1 & 1 & 1 & 1 & 1 & 1 & 1 & 1 & 1 & 1 & 1 & 1 & 1 \\
1 & 1 & 1 & 1 & 1 & 1 & 1 & 1 & 1 & 1 & 1 & 1 & 1 & 1 & 1 & 1 \\
\hline & 1 & 1 & 1 & 1 & 1 & 1 & 1 & 1 & 1 & 1 & 1 & 1 & 1 & 0 & 1
\end{tabular}

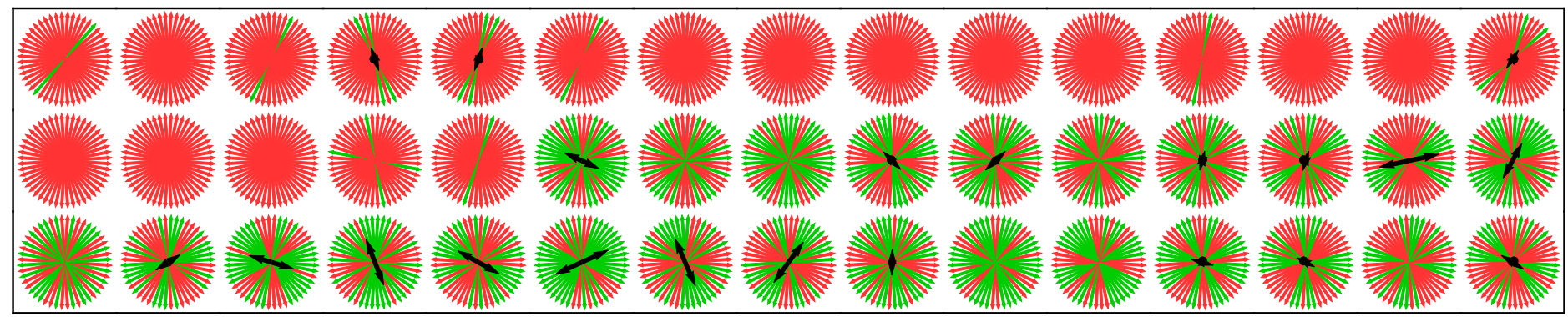

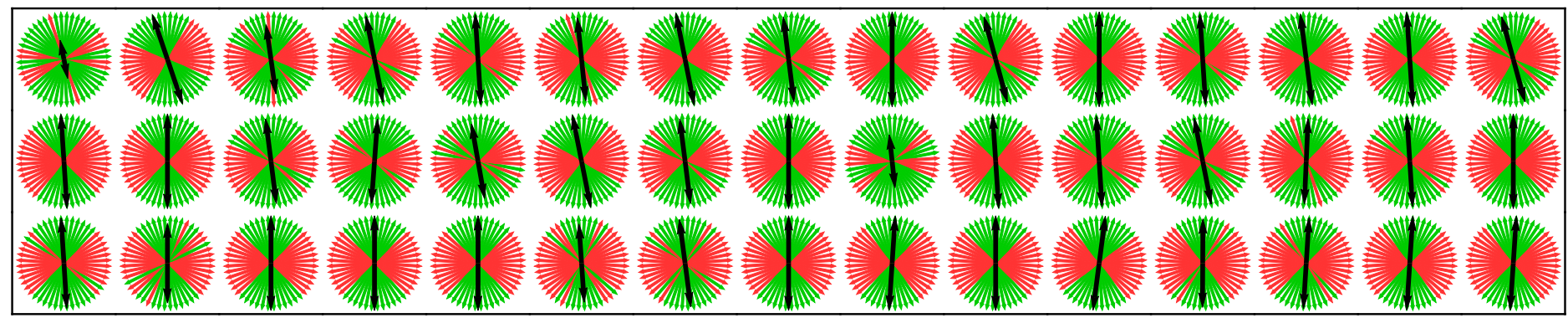
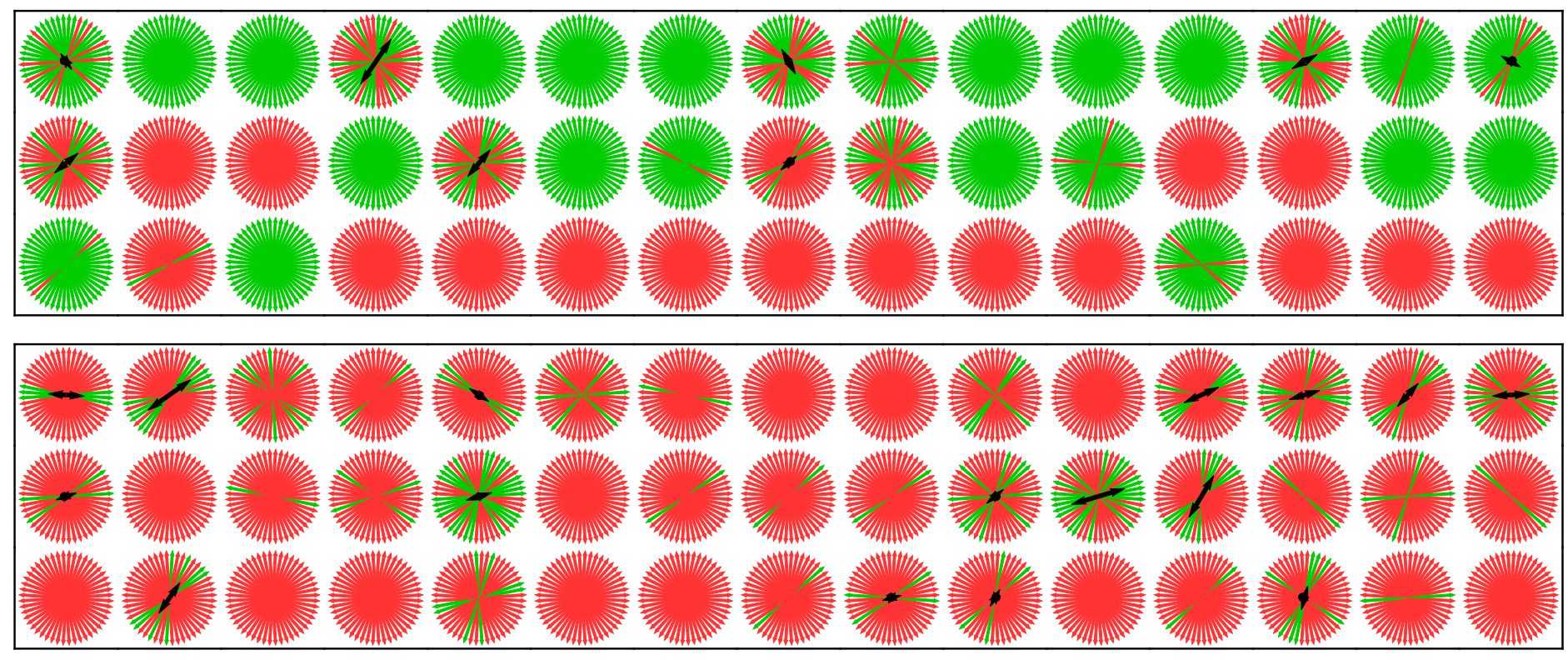

Figure A2: Full times series of all six subjects in Experiment 2. The 45 measurements for each subject (in the temporal order shown by numerals for the first subject) were over a period of 9 hours, and were taken at 12-minute intervals. 

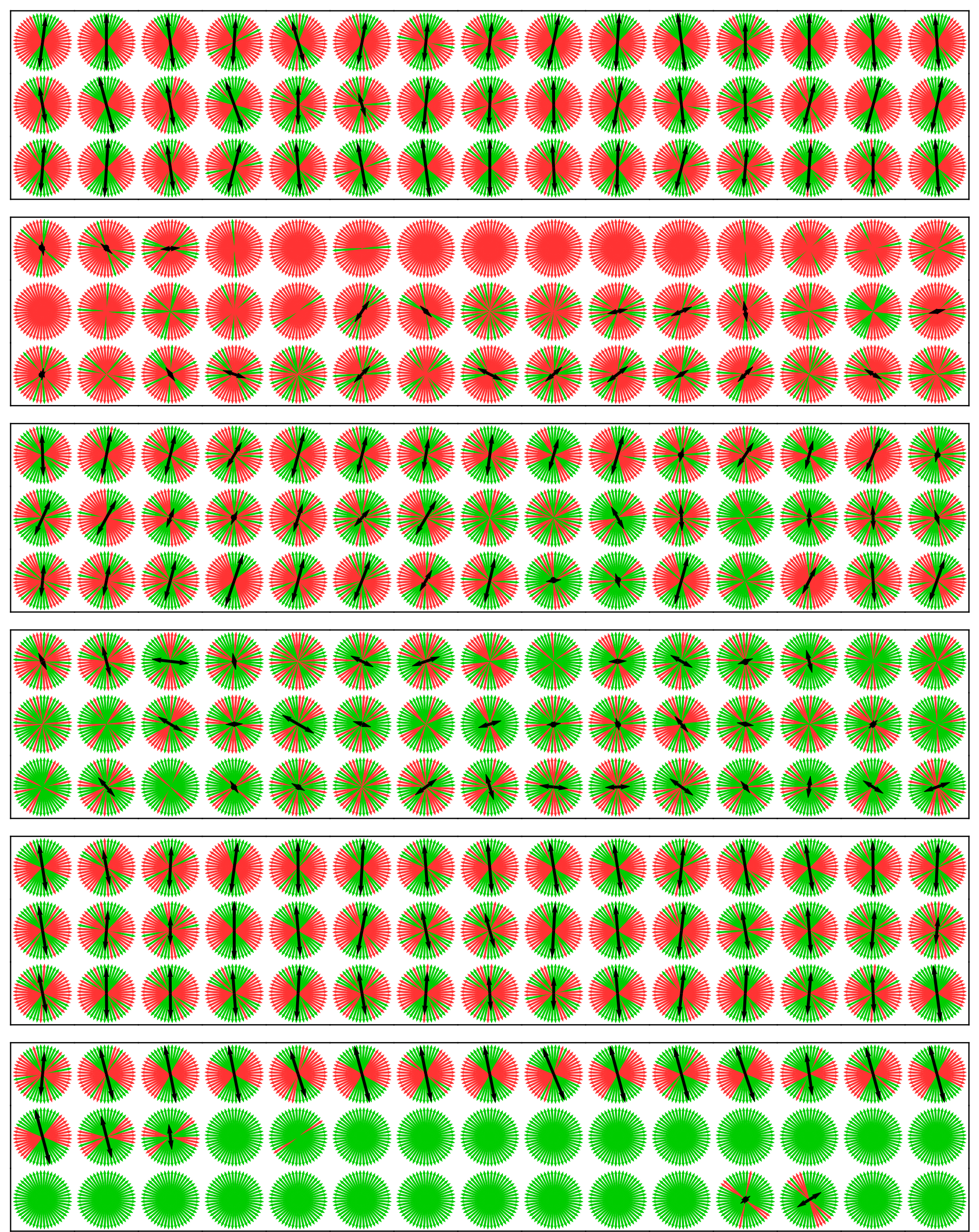

Figure A3: Full times series of all six subjects in Experiment 3. The 45 measurements for each subject (in the temporal order shown by numerals for the first subject) were over a period of 9 hours, and were taken at 12-minute intervals. 


\section{A1 Auxiliary experiment: Temporal threshold for voluntary control}

\section{A1.1 Methods}

The experiment was performed in the laboratory. There were two types of blocks: forced, where the subject was told which motion direction to attempt to perceive, and unforced, with no particular instructions.

\section{A1.1.1 Stimuli}

With the exception of the variable duration of the first frame, the quartet stimuli were identical to those in Experiment 2. The duration of the first frame was 200, 400, 600, 800 or $1000 \mathrm{~ms}$, with the duration of the second frame fixed at $400 \mathrm{~ms}$ as in Experiment 2. As in Experiment 2, each block consisted of 24 trials, sampling stimulus orientations between $0^{\circ}$ and $180^{\circ}$.

On forced blocks, the forced direction of motion (vertical, horizontal, clockwise or counterclockwise) was shown using a visual icon before the beginning of each block. Subjects were subsequently reminded of the forced direction using a verbal auditory cue before the start of each trial, the SOA between the auditory cue and the onset of the first visual frame being $700 \mathrm{~ms}$. Before the start of unforced blocks, subjects were told that there were no particular instructions, and to just report what the motion that they saw; no auditory cues were given.

\section{A1.1.2 Procedure}

All conditions (motion direction to be forced, duration of first frame) were blocked. Each forced block was preceded by an unforced block.

The main part of the experiment consisted of 20 unforced-forced block pairs. Each of the 20 forced blocks has one of the four forced directions and one of the five initial frame durations. This factorial design was randomized, subject to the constraint that no two adjacent forced blocks have the same forced direction.

The main part of the experiment was preceded by the practice conditions, consisting of 4 unforced-forced block pairs. During the practice trials the forced direction on the first forced block was randomly chosen from clockwise or counterclockwise, while the direction on the second block was randomly chosen from vertical or horizontal, with the duration of the first frame equal to $1000 \mathrm{~ms}$ on both blocks. The second two forced blocks were the same as the first two, except with the duration of the first frame equal to $200 \mathrm{~ms}$. Data from the practice conditions was not included in the analysis.

Thus, in total, the experiment consisted of 48 blocks. The blocks succeeded one another so that the each block began 4 minutes after the start of the previous block. Mean duration of each block turned out to be about 70 seconds, so that subjects had, on the average, almost 3 minutes of rest between blocks. 


\section{A1.1.3 Subjects}

Eleven naive subjects, none of whom participated in any other experiment in this study, took part in this experiment. They were paid $10 € /$ hour.

\section{A1.2 Results}

The goal of this experiment was to measure the effectiveness of voluntary effort in modifying each subject's individual bias pattern in the motion quartet. Using the data from the unforced blocks preceding the forced blocks, I took careful precautions to make sure that the underlying, unforced bias pattern immediately preceding the forced block was strong and stable. It was also important to exclude conditions in which the subject's own bias pattern was being forced.

First of all, I excluded from further analysis subjects who had bias patterns that were weak or unstable during the 20 unforced blocks. There were 4 such unstable subjects, which left 7 subjects for the analysis. The dominant bias pattern of 6 of these subjects was vertical, and of the remaining subject counterclockwise.

Each trial of the remaining data was scored either 0 or 1 on four criteria: vertical, horizontal, clockwise and counterclockwise. For the clockwise and counterclockwise criteria, I simply used the responses (which were clockwise or counterclockwise). For the vertical and horizontal criteria, the trial was scored as 1 if the reported motion direction was within $45^{\circ}$ of vertical or horizontal, respectively, and 0 otherwise. Taking the mean of these four scores yielded, for each block, four scores for verticalness, horizontalness, clockwise-ness and counterclockwise-ness.

Even in the 7 subjects with strong and stable biases, there were some fluctuations in the biases. I therefore found unforced blocks in which the mean score for the subject's dominant bias pattern fell below 0.75 . There were 13 such blocks out of a total of 140 . Data from the forced block following these blocks were excluded.

Finally, it is meaningless to test the effectiveness of the instruction, say, to perceive vertical motion for a subject whose dominant bias pattern is already vertical. Therefore, all data where the forced motion was equal to the subject's dominant bias pattern were excluded.

In the remaining data, for each forced block I calculated the mean criterion score for the forced motion, subtracting the same score from the preceding unforced block, considered as a baseline. For example, for a block in which the instruction was to perceive clockwise motion, I calculated the mean clockwise score ( 1 for clockwise responses, 0 for counterclockwise) for that block, and subtracted the same thing for the preceding unforced block. This yields an index for the effectiveness of the voluntary effort, positive if effective and zero otherwise.

The results are shown in Figure A4, which displays the index for the effectiveness of the voluntary effort as a function of the duration of the first frame. As can be seen in the graph, the effectiveness of voluntary effort rises with increasing first-frame duration. The slopes of the effectiveness versus duration are positive in 6 out of 7 subjects, with the mean slope significantly positive $\left(t_{6}=3.24, p=0.02\right)$. $\mathrm{T}$ tests for individual values of the 


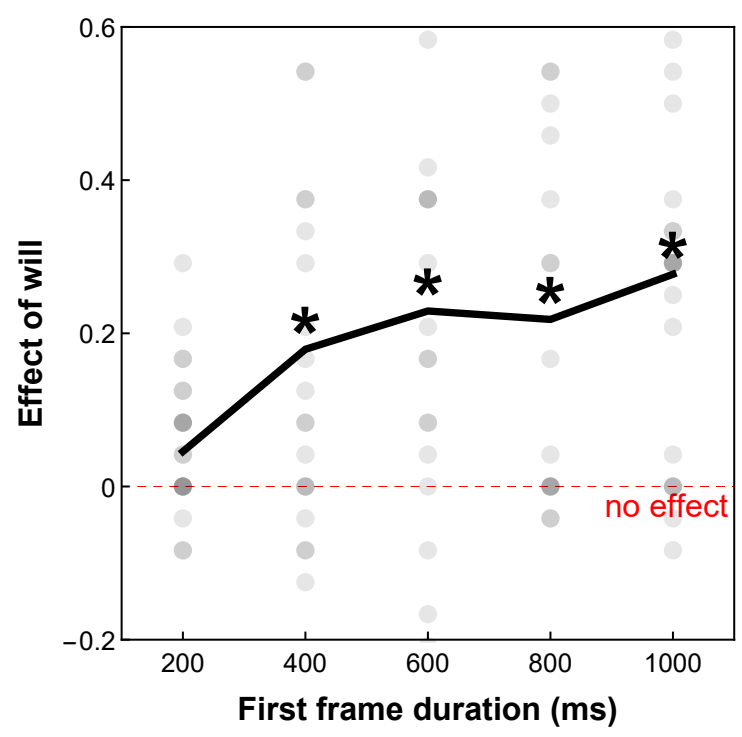

Figure A4: The results of the auxiliary experiment on the effectiveness of voluntary effort to perceive particular motion in the quartet. The horizontal axis represents the duration of the first frame of the quartet, while the vertical axis represents an index of the effectiveness of the voluntary effort, positive if the effort is successful (see text for definition). Dots show data for individual subjects, the solid line the mean over the subjects. Asterisks mark the durations of the first frame for which the effect of voluntary effort is significantly above zero.

duration revealed that the effectiveness is significantly positive for all durations greater than or equal to $400 \mathrm{~ms}$, but not for $200 \mathrm{~ms}$.

\section{A1.3 Discussion}

This experiment has shown that voluntary efforts to control the direction of motion of the quartet become less effective as the duration of the first frame decreases. At duration $200 \mathrm{~ms}$, the effectiveness is close to zero and statistically no different from zero. This is in reasonable agreement with the observation of Ramachandran and Anstis that a cycling (rather than a single-shot) quartet becomes impossible to control voluntarily at temporal frequencies above $3 \mathrm{~Hz}$. It is of course possible that subjects simply reported the direction of motion we instructed them to attempt to perceive, rather than the direction of motion that they actually saw. This would artificially inflate the effectiveness, and therefore would decrease the temporal threshold of voluntary control. Thus, the 200-400 $\mathrm{ms}$ threshold found here should be thought of a lower limit on the actual threshold. 